\title{
Análise da evolução, impacto e formação de redes nos cinco anos do BraSNAM
}

\author{
Luciano A. Digiampietri ${ }^{1}$, Rogério Mugnaini ${ }^{2}$, José J. Pérez-Alcázar ${ }^{1}$, \\ Karina V. Delgado ${ }^{1}$, Esteban F. Tuesta ${ }^{1}$, Jesús P. Mena-Chalco ${ }^{3}$ \\ ${ }^{1}$ Escola de Artes, Ciências e Humanidades da Universidade de São Paulo \\ ${ }^{2}$ Escola de Comunicações e Artes da Universidade de São Paulo \\ ${ }^{3}$ Centro de Matemática, Computação e Cognição da Universidade Federal do ABC
}

digiampietrieusp.br

\begin{abstract}
The Brazilian Workshop on Social Network Analysis and Mining (BraSNAM) is the first specific Brazilian event in the area of social networks analysis and mining. This is an interdisciplinary event and its goal is to integrate the scientific community that research in the area, disseminating the research performed, and enhancing new collaborations. In 2016, the event completed five years. 149 papers were presented as full or short papers, involving 335 authors. In this work, based on the five editions of BraSNAM, we present a quantitative analysis on: (i) bibliographic production, and (ii) the evolution of its co-authorship social network.
\end{abstract}

Resumo. O Brazilian Workshop on Social Network Analysis and Mining (BraSNAM) é o primeiro evento brasileiro específico na área de mineração e análise de redes sociais. Este evento tem natureza interdisciplinar e seu objetivo é integrar a comunidade científica que atua na área, disseminando as pesquisas realizadas e potencializando novas colaborações. Em 2016, o evento completou cinco anos e nele foram apresentados 149 trabalhos referentes a artigos completos ou curtos e que envolveram 335 autores. Neste trabalho, baseados nas cinco edições do BraSNAM, apresentamos uma análise quantitativa sobre: (i) a produção bibliográfica, e (ii) a evolução de sua rede social de coautoria.

\section{Introdução}

A mineração e análise de redes sociais é uma área interdisciplinar que atualmente envolve principalmente as áreas de ciências sociais aplicadas e ciência da computação.

Uma rede social é uma estrutura composta por indivíduos que são conectados por relações. O conceito de redes sociais existe há séculos nas áreas de sociologia e antropologia social, mas ganhou muito destaque nas últimas décadas com o desenvolvimento de métodos computacionais para a análise de redes, bem como a grande disponibilização de informações na web e, em especial, com o surgimento das redes sociais online [Lemieux and Ouimet 2008].

A análise de redes sociais pode ser utilizada para uma grande gama de aplicações, desde a caracterização de comunidades, recomendação de itens, sugestão de relacionamentos, predição de tendências, análise de influências, entre tantas outras [Wasserman and Galaskiewicz 1994, Wasserman and Faust 2009]. 
Redes sociais buscando compreender a estrutura intelectual da informática biomédica analisando tópicos de pesquisa publicados em eventos acadêmicos foram utilizadas em [Jeong and Kim 2010]. Segundo os autores, a análise da literatura publicada em anais de eventos apresenta vantagens sobre abordagens bibliométricas mais comuns, pelo fato de não dependerem do atraso causado pelas bases de dados bibliográficas. Além disso, tal fonte é propícia para análise de domínio, permitindo identificar novos tópicos emergentes e, consequentemente, uma melhor compreensão da área. Adicionalmente, a utilização de análise de redes sociais para definição de links entre os artigos viabiliza a análise de domínio, que usualmente dependia de citações, o que fazia com que estudos só fossem viáveis após considerável intervalo de tempo após o ano de publicação.

Em 2012 ocorreu a primeira edição do Brazilian Workshop on Social Network Analysis and Mining (BraSNAM), o primeiro evento brasileiro específico para tratar assuntos ligados à mineração e análise de redes sociais. O workshop objetiva ser um "facilitador no reconhecimento dos trabalhos desenvolvidos atualmente no país, na troca de conhecimento e na criação de novas colaborações nacionais e internacionais. A ideia do evento é ser multidisciplinar, integrando todos que trabalham ou tenham interesse na área." 1 .

O evento é realizado anualmente e aceita a submissão de artigos longos (12 páginas) e artigos curtos (6 páginas). Cada artigo passa por um processo de revisão por pares às cegas no qual os autores e os revisores desconhecem quem é o avaliador e o avaliado (double blind). Ao longo de seus cinco anos de existência, 149 artigos foram apresentados no BraSNAM e estes contaram com 335 autores diferentes.

O objetivo deste artigo é realizar uma análise da produção bibliográfica e de redes sociais de coautorias considerando as cinco primeiras edições do BraSNAM (de 2012 a 2016). Neste trabalho são levantadas medidas bibliométricas relacionadas ao número de artigos publicados, autores e citações. Adicionalmente, a evolução da rede social de coautorias é analisada observando-se diferentes métricas de rede, bem como a capacidade do evento em agregar novos autores a cada uma de suas edições. Por fim, uma breve análise sobre os títulos das publicações é realizada verificando-se as expressões mais frequentes, os tipos de desafios tratados e as fontes de dados utilizadas.

Acreditamos que o mapeamento aqui realizado permite evidenciar de forma quantitativa características e padrões importantes sobre o BraSNAM. O que o evento publica? Qual o impacto, em termos de citações, dos artigos publicados? Existem comunidades de pesquisadores que participam de forma constante? Quais os tópicos mais pesquisados em todas as edições do evento? Essas são questões que abordamos e discutimos neste trabalho.

\section{Materiais e Métodos}

Neste trabalho foram utilizadas informações de duas fontes diferentes. A primeira corresponde aos sítios web das cinco primeiras realizações do BraSNAM (de 2012 a 2016). Destes sítios foram copiadas as listas de trabalhos longos e curtos que foram apresentados em cada uma das edições do evento. Para o presente trabalho, foram utilizados, para cada

\footnotetext{
${ }^{1}$ BraSNAM: http://www.imago.ufpr.br/csbc2012/anais_csbc/eventos/brasnam. Último acesso em 5 de março de 2017
} 
artigo: o título, a lista de autores e o ano em que foi apresentado. Este processo resultou na identificação de 149 trabalhos e 335 autores.

A segunda fonte de informação foi o Google Acadêmico $^{2}$, utilizado para a obtenção do número de citações recebidas por todos os trabalhos apresentados no BraSNAM. Esta obtenção foi realizada por meio de uma ferramenta desenvolvida para consultar o título de cada artigo no Google Acadêmico e verificar se o título encontrado corresponde ao título procurado. Ao todo, esta abordagem permitiu encontrar 226 citações a trabalhos apresentados no BraSNAM.

Com base nessas informações, foram realizados dois tipos de análise: (i) A análise da produção bibliográfica relacionada com a quantidade de artigos publicados, distribuição dos artigos por autores, distribuição dos autores por artigo, citações recebidas pelos artigos e pelos autores e índices g e h do evento. (ii) A análise de redes sociais através de redes de coautoria criadas para cada ano do evento e também redes acumuladas contendo os dois, os três, os quatro e os cinco primeiros anos do BraSNAM. Para estas redes foram analisadas 14 métricas [Wasserman and Faust 2009] que serviram tanto para caracterizar as redes quando para analisar a evolução da coautoria ao longo dos anos. Adicionalmente, os títulos das publicações foram analisados e foi gerada uma nuvem de palavras e expressões e uma rede de palavras. A discussão dos resultados é descrita na próxima seção.

\section{Resultados}

Ao longo de suas cinco primeiras edições, 149 trabalhos foram apresentados no BraSNAM, sendo 40 trabalhos em 2012, 28 em 2013, 31 em 2014, 32 em 2015 e 18 em 2016.

Dos 149 trabalhos apresentados no evento, 59 receberam uma ou mais citações ${ }^{3}$, totalizando 226 citações. Segundo estes dados, o índice-h do BraSNAM é igual a 7 e o índice-g é igual a 11 [Egghe 2006]. A Figura 1 apresenta o número de artigos de acordo com as citações recebidas. Observa-se que cinco artigos receberam mais de 10 citações, enquanto que 90 ainda não receberam nenhuma e 26 foram citados uma única vez.

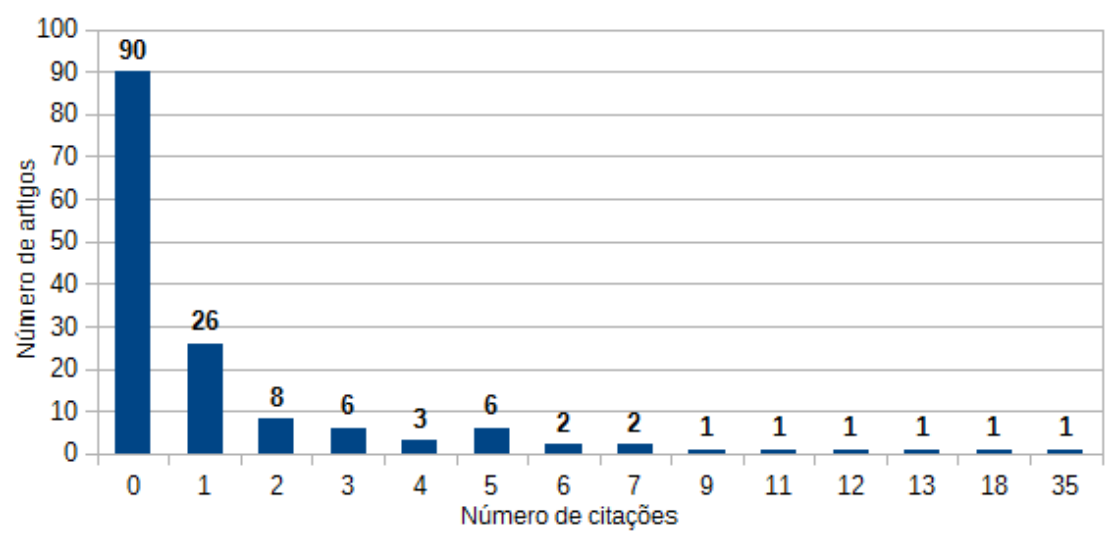

Figura 1. Número de artigos de acordo com as citações recebidas.

\footnotetext{
${ }^{2}$ Google Acadêmico: scholar.google.com.br. Último acesso em 5 de março de 2017.

${ }^{3}$ Dados obtidos do Google Acadêmico em 24/02/2017.
} 
A Tabela 1 contém a referência, o título, o ano do evento e a quantidade de citações recebidas pelos 10 artigos mais citados. Observa-se que estes 10 artigos foram apresentados nas duas primeiras edições do evento, o que não é uma surpresa já que artigos mais antigos possuem mais tempo para serem citados. Estes artigos somam 124 citações o que corresponde a 55\% do total de citações recebidas pelos artigos do BraSNAM.

Tabela 1. Artigos mais citados do BraSNAM.

\begin{tabular}{|c|l|c|c|}
\hline \multicolumn{1}{|c|}{ Referência } & Título An & Cits. \\
\hline [Digiampietri et al. 2012a] & $\begin{array}{l}\text { Minerando e Caracterizando Dados de } \\
\text { Currículos Lattes }\end{array}$ & 2012 & 35 \\
\hline [Digiampietri et al. 2012b] & $\begin{array}{l}\text { Dinâmica das Relações de Coautoria nos Pro- } \\
\text { gramas de Pós-Graduação em Computação no } \\
\text { Brasil }\end{array}$ & 2012 & 18 \\
\hline [Mena-Chalco et al. 2012] & $\begin{array}{l}\text { Caracterizando as redes de coautoria de } \\
\text { currículos Lattes }\end{array}$ & 2012 & 13 \\
\hline [Nascimento et al. 2012] & $\begin{array}{l}\text { Análise de sentimento de tweets com foco em } \\
\text { notícias }\end{array}$ & 2012 & 12 \\
\hline [Gonçalves et al. 2013] & $\begin{array}{l}\text { O que Tweets Contendo Emoticons Podem Re- } \\
\text { velar Sobre Sentimentos Coletivos? }\end{array}$ & 2013 & 11 \\
\hline [do Vale Cunha et al. 2013] & $\begin{array}{l}\text { Redes de títulos de artigos científicos variáveis } \\
\text { no tempo }\end{array}$ & 2013 & 9 \\
\hline [Tuesta et al. 2012] & $\begin{array}{l}\text { Análise temporal da relação orientador- } \\
\text { orientado: um estudo de caso sobre a produti- } \\
\text { vidade dos pesquisadores doutores da área de } \\
\text { Ciência da Computação }\end{array}$ & 2012 & 7 \\
\hline [Digiampietri et al. 2013] & $\begin{array}{l}\text { Predição de coautorias em redes sociais } \\
\text { acadêmicas: um estudo exploratório em Ciência } \\
\text { da Computação }\end{array}$ & 2013 & 7 \\
\hline [Ströele et al. 2012] & $\begin{array}{l}\text { Análise de Redes Sociais Científicas: Modela- } \\
\text { gem }\end{array}$ & $\begin{array}{l}\text { Monitoramento das Interações dos Aprendizes } \\
\text { na Rede Social Twitter como Apoio ao Processo } \\
\text { de Mediação Docente }\end{array}$ & 6 \\
\hline
\end{tabular}

Dos 335 autores de trabalhos do BraSNAM, 254 participaram da publicação de um único trabalho no evento e 7 participaram da publicação de mais de cinco trabalhos. A Figura 2 apresenta um gráfico de barras com a quantidade de artigos publicados pelos autores.

Na média, cada artigo apresentado no BraSNAM teve 3,4 autores. Ao observar essa medida para cada edição do evento, nota-se que ela oscilou entre 2,7 e 3,8. Já a mediana foi de 3 autores por artigo para todas as edições do evento. Verificou-se uma correlação positiva igual a 0,3 entre o número de coautores e a quantidade de citações recebidas pelos artigos, considerando todas as edições do BraSNAM. Em particular, o maior valor de correlação $(0,59)$ entre o número de coautores e o número de citações recebidas pelos artigos ocorreu na primeira edição do evento.

A Tabela 2 apresenta o número de artigos publicados no BraSNAM, o número de citações recebidas e o número de coautores dos sete autores que publicaram mais de 
cinco artigos ao longo da história do evento. Destaca-se que o Autorl é o que participou da maior quantidade de trabalhos apresentados no BraSNAM (10\% do total) e que recebeu a maior quantidade de citações (41\% do total de citações). Já o Autor2 foi aquele que colaborou com a maior quantidade de autores (cerca de $8,7 \%$ do total de autores).

Tabela 2. Autores que tiveram a maior quantidade de artigos publicados no BraSNAM.

\begin{tabular}{|l|c|c|c|}
\hline Autor & Artigos & Citações & Coautores \\
\hline Autor1 & 15 & 93 & 23 \\
\hline Autor2 & 13 & 23 & 29 \\
\hline Autor3 & 11 & 8 & 19 \\
\hline Autor4 & 8 & 5 & 20 \\
\hline Autor5 & 7 & 20 & 18 \\
\hline Autor6 & 7 & 11 & 15 \\
\hline Autor7 & 6 & 79 & 11 \\
\hline
\end{tabular}

Dos autores que tiveram seus trabalhos apresentados e publicados no BraSNAM, apenas 4 não têm nenhum colaborador/coautor. A quantidade de colaboradores mais frequente é 2 (78 autores possuem 2 colaboradores) e em segundo lugar está 3 (69 autores possuem três colaboradores), conforme pode ser observado na Figura 3.

Com base na coautoria dos trabalhos apresentados no BraSNAM foram geradas

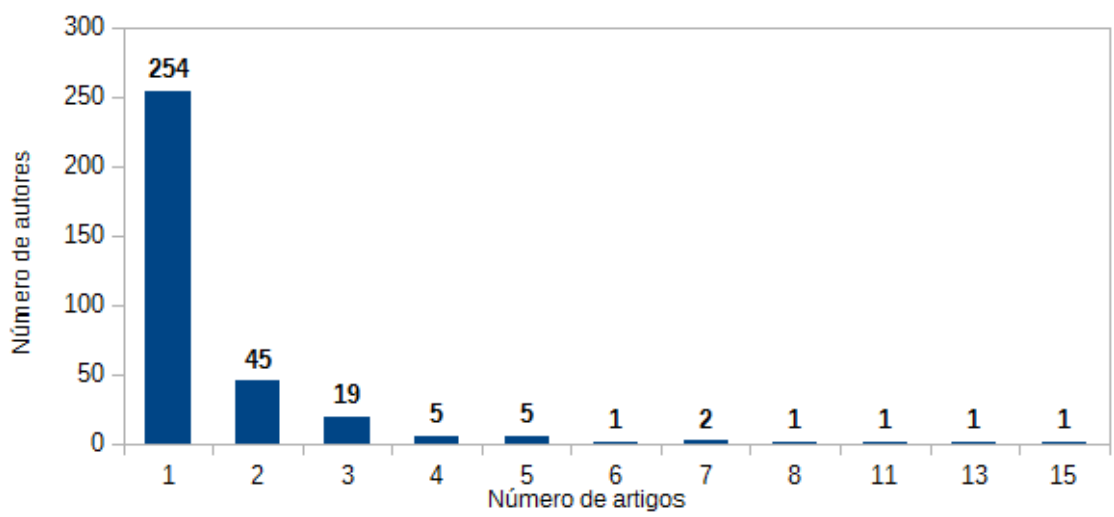

Figura 2. Quantidade de artigos publicados no BraSNAM pelos autores.

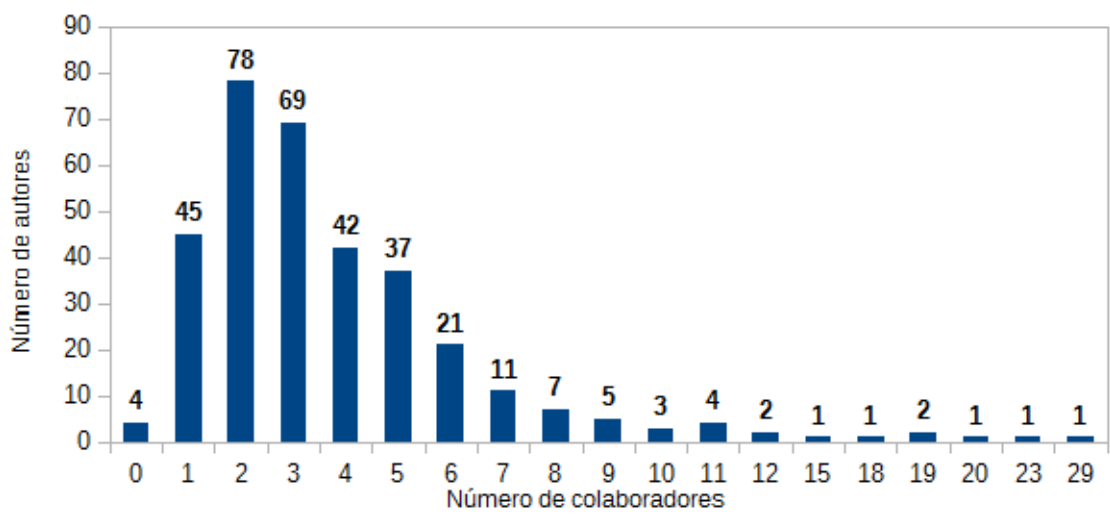

Figura 3. Quantidade de colaboradores dos autores do BraSNAM. 


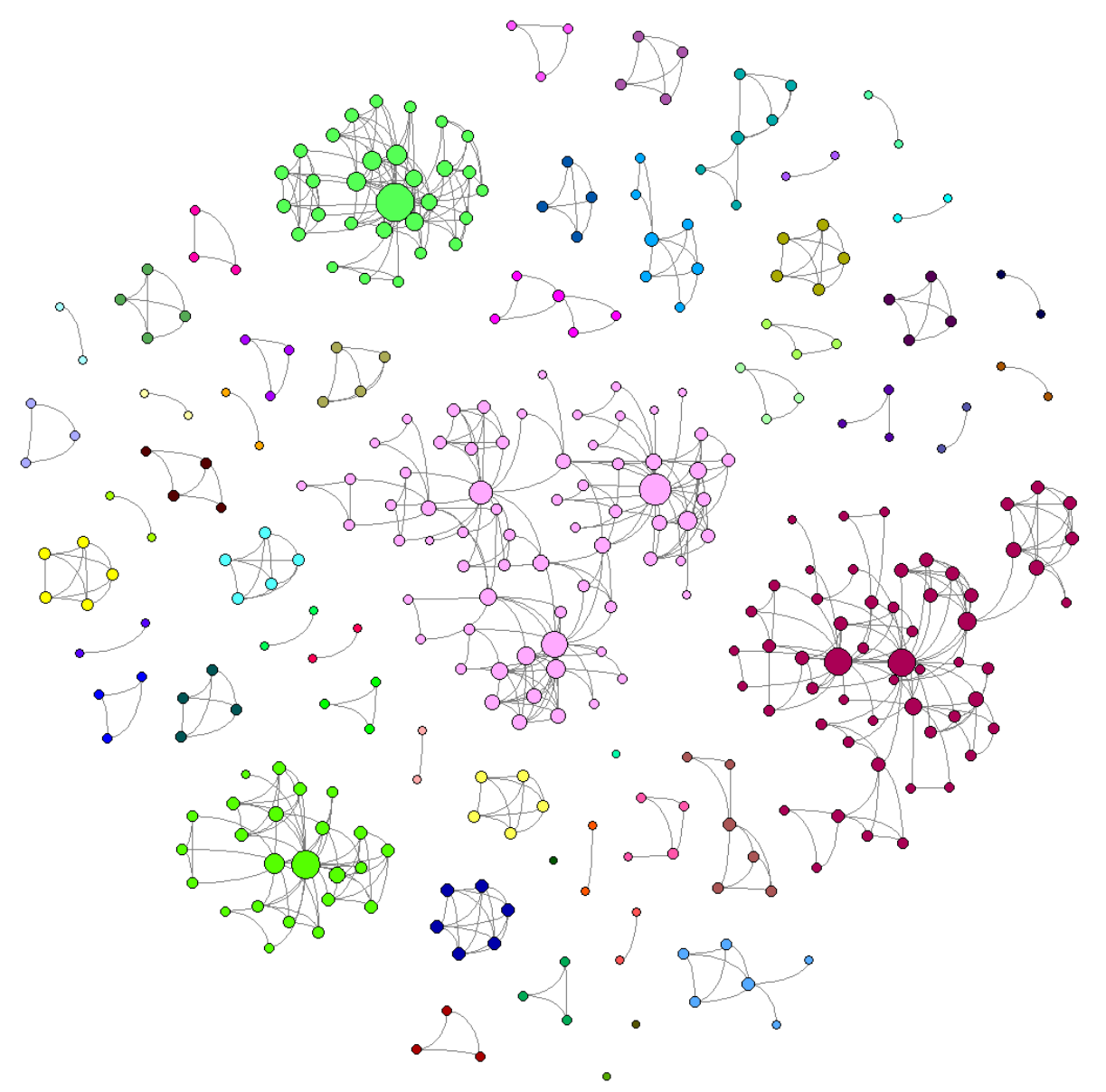

Figura 4. Rede social das coautorias acumuladas de 2012 a 2016.

redes sociais de coautorias. Nestas redes, cada autor corresponde a um nó e as arestas representam a relação de colaboração na produção de ao menos um artigo no BraSNAM. Dessa forma, a coautoria de cada artigo gera uma clique entre os nós coautores.

Ao todo foram geradas nove redes de coautorias, uma para cada ano do BraSNAM (de 2012 a 2016) e as redes com as coautorias acumuladas 2012-2013, 2012-2014, 20122015 e 2012-2016). Apenas para facilitar a visualização das figuras e tabelas a seguir, também é apresentada uma rede com a coautoria "acumulada" de 2012 a 2012, que, de fato, é a própria rede de 2012. Assim, são apresentadas cinco redes anuais e cinco com as coautorias acumuladas.

A Figura 4 apresenta todas as coautorias ocorridas nos trabalhos apresentados no BraSNAM de 2012 a 2016. Cada um dos 335 autores está representado como um nó e as relações de coautorias são representadas por arestas (ao todo, 653 arestas). O tamanho dos nós é proporcional à centralidade de grau.

O Autor2 da tabela 2 corresponde ao maior nó da rede, no componente conexo verde na esquerda superior da Figura 4. Já o Autorl é o maior nó do componente gigante que está no centro da rede.

A rede da Figura 4 é composta por 53 componentes conexos. Há 4 autores individuais (que não estão ligados a nenhum outro). O tamanho do componente mais frequente é 
2 (com 16 componentes desse tamanho) e o maior componente contém 68 autores (20,3\% dos autores da rede). A Figura 5 apresenta a quantidade de componentes para cada quantidade de autores.

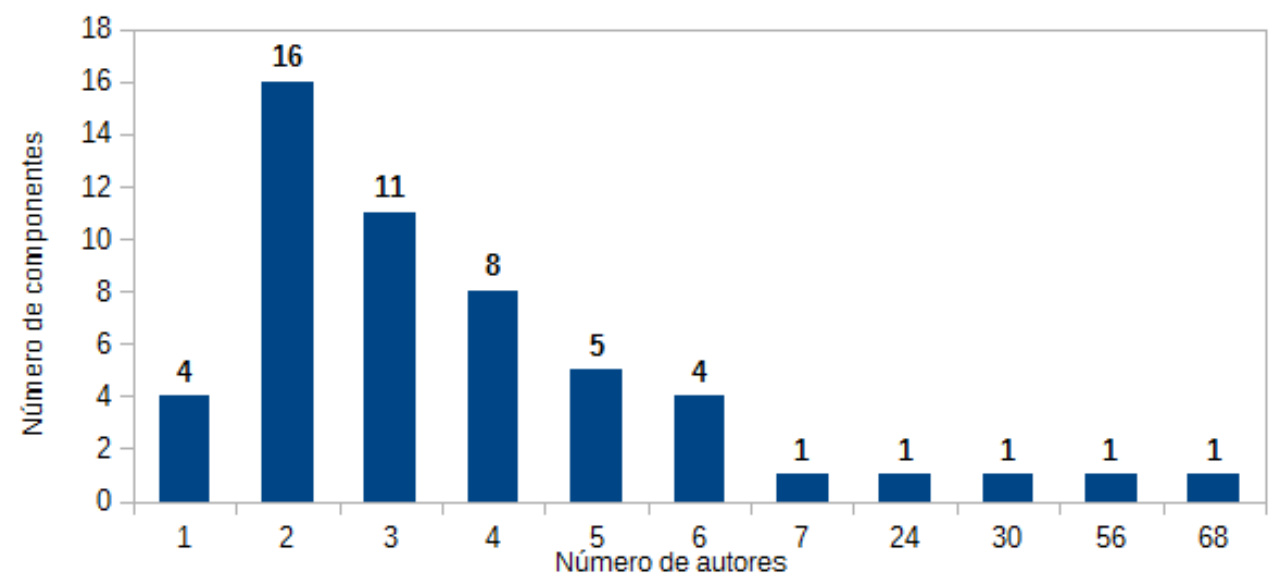

Figura 5. Número de autores por componente conexo - rede acumulada 20122016

A Tabela 3 apresenta algumas métricas de todas as redes produzidas. Observa-se, pelo número de nós a quantidade de autores que tiveram artigos apresentados em cada uma das edições do BraSNAM. O número máximo foi na primeira edição do evento (106, ou seja, 32\% do total acumulado de autores do evento) e o mínimo na última (42). Pelas redes acumuladas, observa-se que a cada edição do BraSNAM novos autores tiveram seus trabalhos apresentados. Além do grande número de autores em 2012, a edição de 2013 atraiu 58 novos autores, além de contar com a participação de 26 que já haviam participado da primeira edição do evento. A edição de 2014 atraiu 75 novos autores, já a edição de 2015 atraiu 66 e a versão de 2016 atraiu 30 novos autores, totalizando 335 autores. A Figura 6 apresenta as redes anuais e as redes acumuladas.

Em termos de densidade, todas as redes são pouco densas (densidades abaixo de 0,06 ou seja, menos de $6 \%$ das arestas possíveis). Estes valores baixos são esperados em redes de coautorias, pois a grande maioria dos autores é coautor de um pequeno número de pessoas [Digiampietri et al. 2012a]. A rede mais densa é a rede de 2016 que é justamente a rede com o menor número de autores.

As redes são todas desconexas, assim, algumas medidas só podem ser medidas considerando seus componentes conexos. O diâmetro das redes varia de 2 a 8 . Os maiores diâmetros são encontrados nas redes acumuladas de 2012-2015 e 2012-2016 e ocorrem devido à conexão existente entre diferentes artigos (artigos que possuem um ou mais autores em comum).

Considerando que cada artigo corresponde a uma clique, o tamanho da clique máxima, em redes de coautoria, tipicamente, corresponde ao maior número de autores de um dado artigo. Esta característica ocorre na rede de coautorias do BraSNAM, cuja maior clique tem tamanho 8 e ocorre porque em 2015 há um artigo com 8 autores.

O número de componentes conexos em cada edição do BraSNAM é sempre menor do que o número de artigos, isto ocorre porque em todas as edição há autores comuns entre diferentes artigos, o que une as cliques correspondentes a cada artigo. Considerando as 


\begin{tabular}{|c|c|c|c|c|c|c|c|c|c|c|c|c|c|c|}
\hline & 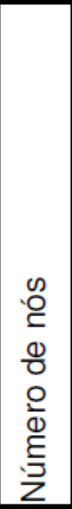 & 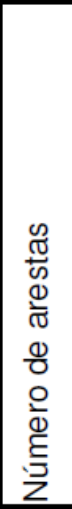 & $\begin{array}{l}\frac{0}{0} \\
\frac{\pi}{0} \\
\frac{0}{02} \\
\frac{2}{0} \\
0\end{array}$ & 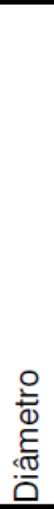 & 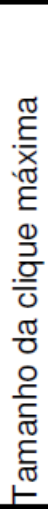 & 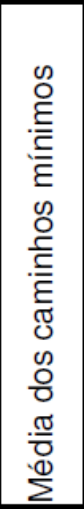 & 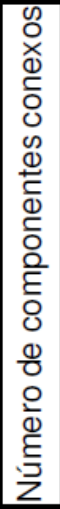 & 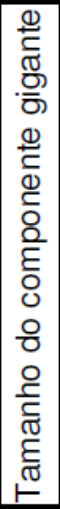 & 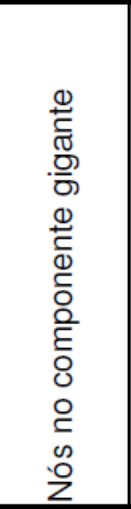 & 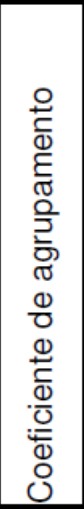 & 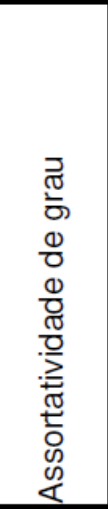 & 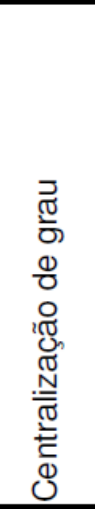 & 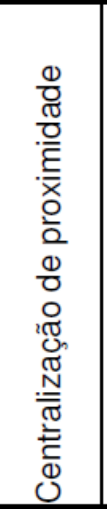 & 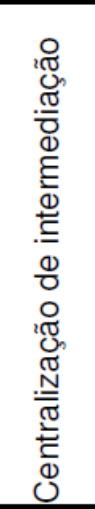 \\
\hline 2012 & 106 & 195 & 0,035 & 5 & 7 & 2,13 & 19 & 31 & $29,25 \%$ & 0,64 & 0,102 & 0,108 & 0,005 & 0,048 \\
\hline 2013 & \begin{tabular}{|l|}
84 \\
\end{tabular} & 148 & 0,042 & 4 & 7 & 1,81 & 17 & 18 & $21,43 \%$ & 0,83 & 0,425 & 0,066 & 0,004 & 0,021 \\
\hline 2014 & 96 & 161 & 0,035 & 3 & 6 & 1,45 & 23 & 17 & $17,71 \%$ & 0,78 & 0,199 & 0,133 & 0,003 & 0,020 \\
\hline 2015 & 99 & 176 & 0,036 & 3 & 8 & 1,56 & 22 & 17 & $17,17 \%$ & 0,75 & 0,266 & 0,127 & 0,002 & 0,019 \\
\hline 2016 & 42 & 44 & 0,051 & 2 & 5 & 1,19 & 13 & 5 & $11,90 \%$ & 0,85 & 0,551 & 0,046 & 0,002 & 0,006 \\
\hline 2012-2012 & 106 & 195 & 0,035 & 5 & 7 & 2,13 & 19 & 31 & $29,25 \%$ & 0,64 & 0,102 & 0,108 & 0,005 & 0,048 \\
\hline 2012-2013 & 164 & 320 & 0,024 & 5 & 7 & 2,46 & 24 & 39 & $23,78 \%$ & 0,62 & 0,088 & 0,080 & 0,002 & 0,032 \\
\hline $2012-2014$ & 239 & 464 & 0,016 & 6 & 7 & 2,64 & 38 & 48 & $20,08 \%$ & 0,58 & 0,048 & 0,068 & 0,001 & 0,024 \\
\hline $2012-2015$ & 305 & 616 & 0,013 & 8 & 8 & 2,93 & 46 & 66 & $21,64 \%$ & 0,54 & $-0,001$ & 0,082 & 0,001 & 0,022 \\
\hline $2012-2016$ & 335 & 653 & 0,012 & 8 & 8 & 2,95 & 53 & 68 & $20,30 \%$ & 0,53 & 0,014 & 0,075 & 0,001 & 0,020 \\
\hline
\end{tabular}

Tabela 3. Métricas das redes sociais de coautoria.

redes acumuladas, o número de componentes cresce gradativamente a cada ano, iniciando com 19 componentes em 2012 e terminando com 53 componentes na rede acumulada até 2016. É importante destacar que ao longo dos anos existem componentes conexos que se fundem, mas mesmo assim há mais novos componentes do que fusões (conforme pode ser observado na Figura 6).

O tamanho do componente gigante (maior componente conexo da rede) aumentou gradativamente nas redes acumuladas. Em 2012 esse componente possuía 31 autores, em 2013 esse componente recebeu 8 novos autores. Já em 2014, um novo componente assumiu a função de componente gigante, contendo 48 autores. Em 2015 esse componente se une a outro componente além de receber novos autores, totalizando 66 autores. Por fim, na rede acumulada até 2016, dois novos autores passam a participar do componente, somando 68 autores. Em termos de porcentagem de indivíduos no componente gigante, nas redes de coautorias acumuladas, essa porcentagem variou entre $20 \%$ e $30 \%$. Observa-se com o passar dos anos que autores de diferentes grupos começaram a cooperar, causando a união de diferentes componentes.

O coeficiente de agrupamento ou coeficiente de clusterização mede a transitividade das relações em uma rede. Em redes de coautoria (ou redes de cliques em geral), esse coeficiente costuma ter valores elevados, pois em uma clique esse valor é máximo (isto é, igual a 1,0). O maior valor de coeficiente de clusterização ocorreu na rede de coautorias de $2016(0,85)$. Já a rede de coautorias acumuladas de 2012 a 2016 foi a que apresentou o menor valor para esta métrica $(0,53)$.

A assortatividade de grau mede a tendência de autores que possuem o mesmo grau de se relacionarem. Em redes de coautoria esta medida é importante por indicar se há uma tendência da existência de relações apenas de publicações individuais (pois em cliques 

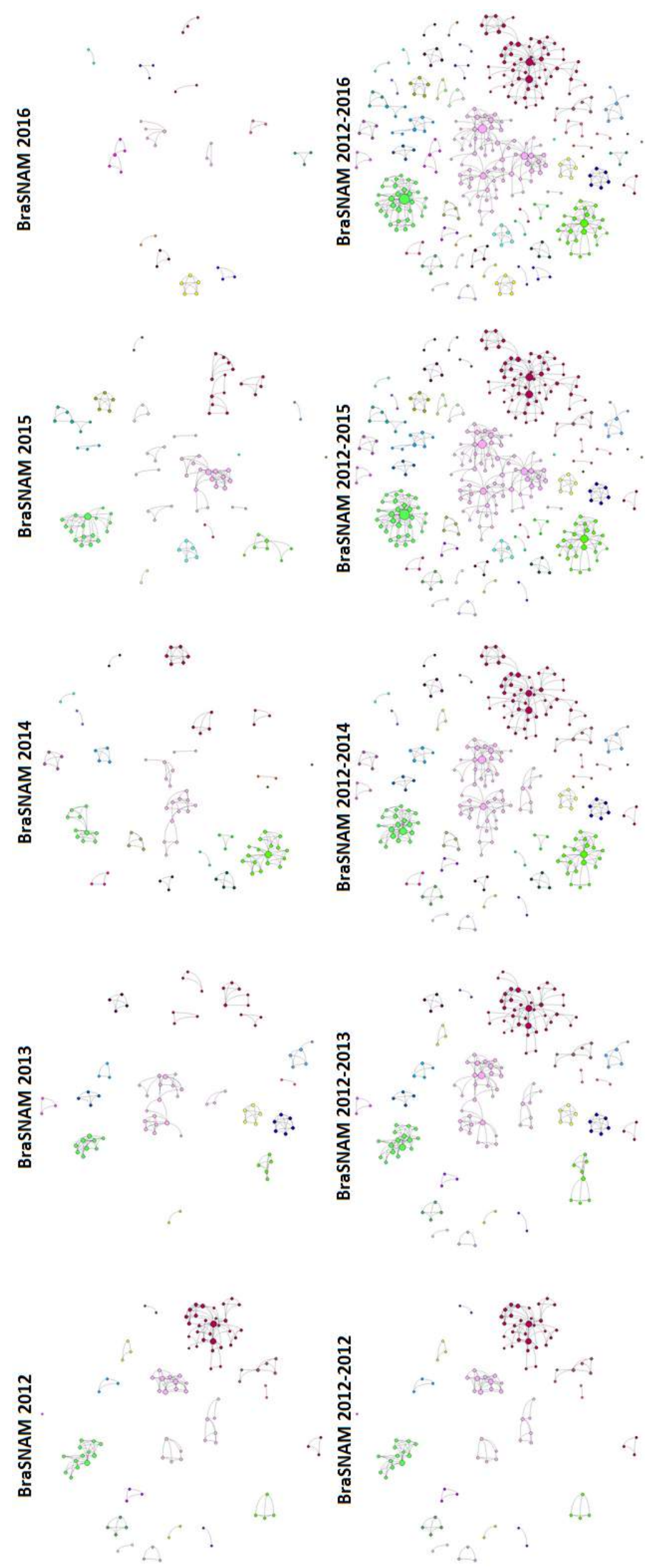

Figura 6. Redes sociais das coautorias e coautorias acumuladas de 2012 a 2016. 
isoladas o valor da assortatividade de grau é máxima, isto é, 1,0, pois todos os autores têm o mesmo grau). A rede de coautorias de 2016 apresentou o maior valor de assortatividade (0,551), já as redes acumuladas de 2012 a 2015 e 2012 a 2016 apresentaram valores muito próximos de zero, indicando que não há uma tendência de autores com mesmo grau de se relacionarem.

As medidas de centralização medem o quão central o nó mais central da rede é em relação aos demais. As três medidas de centralização adotadas, baseadas no grau, na proximidade e na intermediação apresentaram valores não muito elevados o que é positivo por indicar que nenhum autor é extremamente central nestas redes. O maior valor encontrado para a centralização de grau ocorreu em 2014 (0,133). Já a maior centralização de proximidade e de intermediação ocorreram em 2012 (respectivamente, 0,005 e 0,048). A rede acumulada de 2012 a 2016 foi a que apresentou os menores valores de centralização indicando que no conjunto de autores e relações de coautoria de toda a história do BraSNAM a importância dos autores está dividida na rede.

A Figura 7(a) contém a nuvem de palavras e expressões ${ }^{4}$ dos títulos dos 149 trabalhos apresentados no BraSNAM. Aqui, destacam-se inicialmente os termos "redes sociais", "análise redes" e algumas de suas variações. Observam-se também algumas fontes de dados utilizadas como "Twitter" e "Plataforma Lattes". É possível também observar termos relativos ao domínio acadêmico, como "redes coautoria", "pós graduação" e "artigos científicos". Por fim, observa-se alguns termos relacionados ao tipo de problema tratado nos artigos, como "análise sentimentos" e "predição".

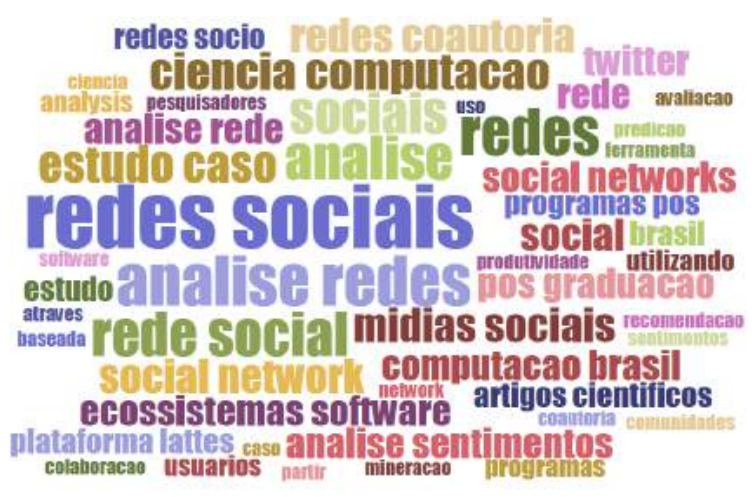

(a) Nuvem de palavras e expressões

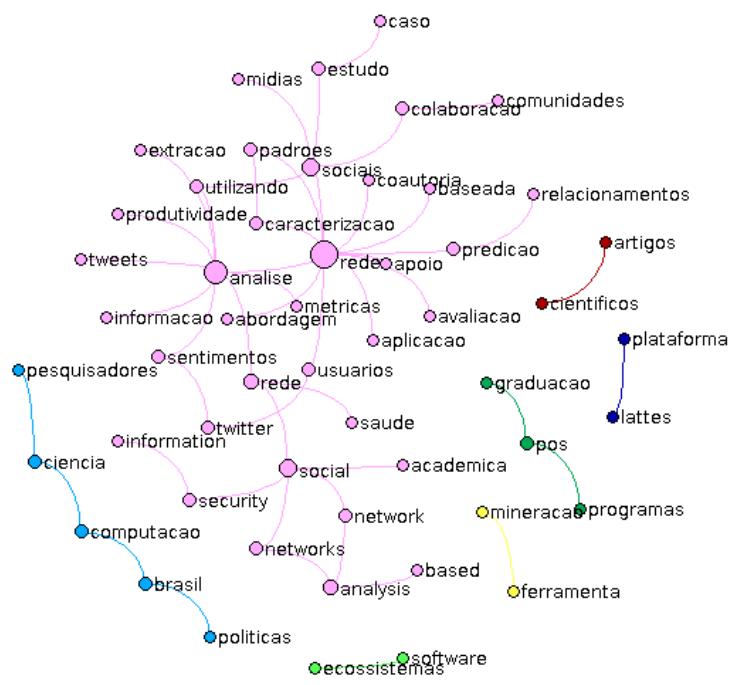

(b) Rede de palavras

Figura 7. Informações extraídas dos títulos dos artigos publicados no BraSNAM.

Já a Figura 7(b) contém a rede das palavras presentes nos títulos. Nesta rede, cada palavra que apareceu ao menos três vezes nos títulos dos trabalhos apresentados no BraSNAM é representada por um nó e uma aresta indica que duas palavras apareceram de forma consecutiva nos títulos ao menos duas vezes. Aqui, destacam-se os sete componentes conexos. Os seis menores são compostos por "ecossistemas software", "pesquisadores ciência computação Brasil políticas", "ferramenta mineração", "programas pós

\footnotetext{
${ }^{4}$ Nuvem gerada pela ferramenta Word Cloud Generator: https://www.jasondavies.com/wordcloud/
} 
graduação", "Plataforma Lattes" e "artigos científicos". Já o componente gigante possui 34 palavras que giram em torno de "análise redes sociais" e suas variações. Exemplos de palavras ligadas neste componente são: "predição relacionamentos", "usuários Twitter", "análise sentimentos", "rede saúde" e "information security".

\section{Considerações Finais}

A mineração e análise de redes sociais é uma área interdisciplinar que ganhou muito destaque nos últimos anos com a grande disponibilização de dados na web e, especialmente, dados oriundos de redes sociais online.

A importância do tema e a visão dos organizadores do BraSNAM em perceber que pesquisas na área estavam tomando corpo no Brasil, mas em iniciativas ainda bastante espalhadas, justificou a criação do Brazilian Workshop on Social Network Analysis and Mining.

Este artigo analisou a rede social formada pelos autores dos trabalhos apresentados nos primeiros cinco anos do BraSNAM, além de realizar uma análise da produção bibliográfica sobre estes trabalho. Observou-se uma evolução do evento que anualmente tem atraído novos pesquisadores para apresentar seus trabalhos, ao mesmo tempo em que os grupos de pesquisadores envolvidos no evento têm se consolidado e expandido.

$\mathrm{O}$ evento cumpre assim seus objetivos, abordando diferentes temas ligados à mineração e análise de redes sociais, aplicados a variados domínios e usando informações de diferentes fontes.

\section{Agradecimentos}

Este trabalho foi parcialmente financiado por CAPES, CNPq e FAPESP.

\section{Referências}

[Digiampietri et al. 2013] Digiampietri, L., Santiago, C., and Alves, C. (2013). Predição de coautorias em redes sociais acadêmicas: um estudo exploratório em Ciência da Computação. In II Brazilian Workshop on Social Network Analysis and Mining (BraSNAM 2013) - Anais do XXXIII Congresso da Sociedade Brasileira de Computação (CSBC 2013), page 12, Maceió, AL, Brasil.

[Digiampietri et al. 2012a] Digiampietri, L. A., Mena-Chalco, J., Perez-Alcazar, J. J., Tuesta, E. F., Delgado, K., and Mugnaini, R. (2012a). Minerando e Caracterizando Dados de Currículos Lattes. In Brazilian Workshop on Social Network Analysis and Mining (BraSNAM 2012) Anais do XXXII Congresso da Sociedade Brasileira de Computação (CSBC 2012), page 12, Curitiba, PR, Brasil.

[Digiampietri et al. 2012b] Digiampietri, L. A., Mena-Chalco, J., Silva, G. S., Oliveira, L., Malheiro, A., and Meira, D. (2012b). Dinâmica das Relações de Coautoria nos Programas de pós-Graduação em Computação no Brasil. In Brazilian Workshop on Social Network Analysis and Mining (BraSNAM 2012) - Anais do XXXII Congresso da Sociedade Brasileira de Computação (CSBC 2012), page 12, Curitiba, PR, Brasil.

[do Vale Cunha et al. 2013] do Vale Cunha, M., Rosa, M. G., de Sousa Fadigas, I., Miranda, J. G. V., and de Barros Pereira, H. B. (2013). Redes de títulos de artigos científicos variáveis no tempo. 
In II Brazilian Workshop on Social Network Analysis and Mining (BraSNAM 2013) - Anais do XXXIII Congresso da Sociedade Brasileira de Computação (CSBC 2013), page 12, Maceió, AL, Brasil.

[Egghe 2006] Egghe, L. (2006). Theory and practise of the g-index. Scientometrics, 69(1):131-152.

[Gonçalves et al. 2013] Gonçalves, P., Benevenuto, F., and de Almeida, V. A. F. (2013). O que Tweets Contendo Emoticons Podem Revelar Sobre Sentimentos Coletivos? In II Brazilian Workshop on Social Network Analysis and Mining (BraSNAM 2013) - Anais do XXXIII Congresso da Sociedade Brasileira de Computação (CSBC 2013), page 12, Maceió, AL, Brasil.

[Jeong and Kim 2010] Jeong, S. and Kim, H.-G. (2010). Intellectual structure of biomedical informatics reflected in scholarly events. Scientometrics, 85(2):541-551.

[Lemieux and Ouimet 2008] Lemieux, V. and Ouimet, M. (2008). Análise Estrutural das Redes Sociais. Instituto Piaget.

[Mena-Chalco et al. 2012] Mena-Chalco, J., Digiampietri, L., and Jr., R. C. (2012). Caracterizando as redes de coautoria de currículos Lattes. In Brazilian Workshop on Social Network Analysis and Mining (BraSNAM 2012) - Anais do XXXII Congresso da Sociedade Brasileira de Computação (CSBC 2012), page 12, Curitiba, PR, Brasil.

[Nascimento et al. 2012] Nascimento, P., Aguas, R., de Lima, D., Kong, X., Osiek, B., Xexéo, G., and de Souza, J. (2012). Anlíise de sentimento de tweets com foco em notícias. In Brazilian Workshop on Social Network Analysis and Mining (BraSNAM 2012) - Anais do XXXII Congresso da Sociedade Brasileira de Computação (CSBC 2012), page 12, Curitiba, PR, Brasil.

[Oliveira et al. 2012] Oliveira, R. F., Araújo, J., Medeiros, F. P., and Brito, A. V. (2012). Monitoramento das Interações dos Aprendizes na Rede Social Twitter como Apoio ao Processo de Mediação Docente. In Brazilian Workshop on Social Network Analysis and Mining (BraSNAM 2012) - Anais do XXXII Congresso da Sociedade Brasileira de Computação (CSBC 2012), page 12, Curitiba, PR, Brasil.

[Ströele et al. 2012] Ströele, V., ao, G. Z., and Souza, J. M. (2012). Análise de Redes Sociais Científicas: Modelagem. In Brazilian Workshop on Social Network Analysis and Mining (BraSNAM 2012) - Anais do XXXII Congresso da Sociedade Brasileira de Computação (CSBC 2012), page 12, Curitiba, PR, Brasil.

[Tuesta et al. 2012] Tuesta, E. F., Delgado, K. V., Digiampietri, L. A., Alcazar, J. J. P., Mugnaini, R., and Mena-Chalco, J. (2012). Análise temporal da relação orientador-orientado: um estudo de caso sobre a produtividade dos pesquisadores doutores da área de Ciência da Computação. In Brazilian Workshop on Social Network Analysis and Mining (BraSNAM 2012) - Anais do XXXII Congresso da Sociedade Brasileira de Computação (CSBC 2012), page 12, Curitiba, PR, Brasil.

[Wasserman and Faust 2009] Wasserman, S. and Faust, K. (2009). Social network analysis: methods and applications. Structural analysis in the social sciences. Cambridge University Press, 19 edition.

[Wasserman and Galaskiewicz 1994] Wasserman, S. and Galaskiewicz, J. (1994). Advances in social network analysis research in the social and behavioral sciences. SAGE Focus Editions. SAGE Publications. 